International Journal of Advanced Academic Studies 2020; 2(3): 706-708

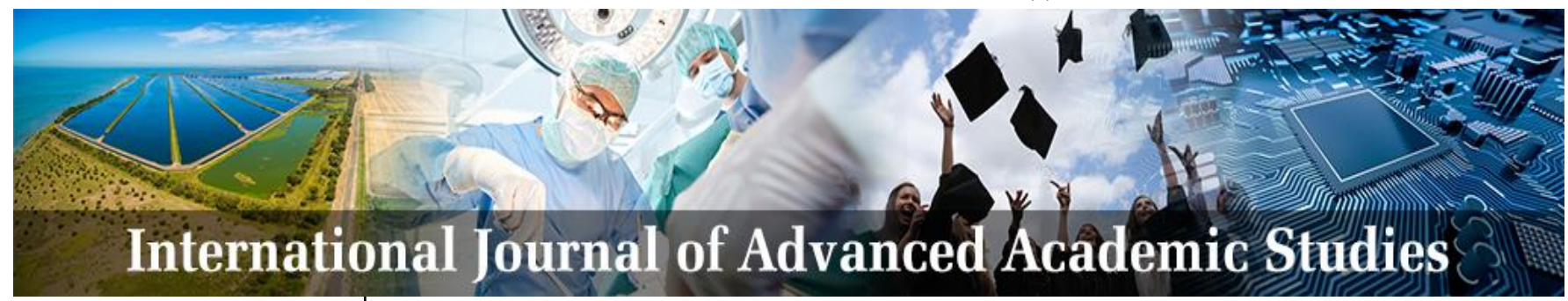

E-ISSN: 2706-8927

P-ISSN: 2706-8919

www.allstudyjournal.com IJAAS 2020; 2(3): 706-708

Received: 17-05-2020

Accepted: 21-08-2020

Namrata Kumari

Department of Psychology,

Lalit Narayan Mithila

University, Darbhanga, Bihar,

India
Corresponding Author: Namrata Kumari

Department of Psychology,

Lalit Narayan Mithila

University, Darbhanga, Bihar,

India

\section{Religious coping strategies for relieving stress}

\section{Namrata Kumari}

DOI: $\underline{\text { https://doi.org/10.33545/27068919.2020.v2.i3j.273 }}$

\begin{abstract}
The study was conducted to assess the effect of reciting "OM" word on relieving perceived stress of 30 married adults ( 20 males and 10 females) aged between 25 to 35 years. On the basis of Perceived Stress scale (Cohen, 1988) high stressed subjects were screened and they were asked to recite OM word daily in the morning for 10 minutes with intention and devotion and after that they were asked to relax for 10 minutes in a garden under the supervision of a yoga teacher for 21 days. After the session was over subjects' perceived stress was assessed and the mean scores of first test and the subsequent test were compared through t-ratio statistical significance test. Results showed that after recital of OM sound with intention and devotion the level of perceived stress was reduced significantly.
\end{abstract}

Keywords: OM, married adults, stress

\section{Introductions}

Religious practices in different forms like meditation, prayer, reki, touch, mantra recitation and the like, for good health and relief from illness have been in practice for millennia in different cultures. There is a description in Bible (21:21) that "Faith can move mountains Different types of meditation have been shown to result in psychological and biological changes that are actually or potentially associated with improved health. Meditation has been found to produce a clinically significant reduction in resting as well as ambulatory blood pressure (Barnes et al., 2004; Kryscio, 2008) ${ }^{[19]}$, to reduce heart rate (Solberg et al., 2004) ${ }^{[23]}$, to result in cardiorespiratory synchronization (CysarzandB?ssing, 2005), to alter levels of melatonin and serotonin (Solberg et al., 2004) ${ }^{[24]}$ to suppress corticostriatalglutamatergic neurotransmission (Kjaer et al., 2002) ${ }^{[18]}$, to boost the immune response (Davidson et al., $2003)^{[8]}$. To decrease the levels of reactive oxygen species as measured by ultraweak photon emission (Van Wijk et al., 2008) ${ }^{[28]}$, to reduce stress and promote positive mood states (Jain et al., 2007) ${ }^{[13]}$, to reduce anxiety and pain and enhance self-esteem (Bonadonna, 2003), and to have a favorable influence on overall and spiritual quality of life in late-stage disease (Williams et al., 2005) ${ }^{[30]}$. Interestingly, spiritual meditation has been found to be superior to secular meditation and relaxation in terms of decrease in anxiety and improvement in positive mood, spiritual health, spiritual experiences and tolerance to pain (Wachholtz and Pargament, 2005) ${ }^{[29]}$.

Prayer is a special form of meditation and may therefore convey all the health benefits that have been associated with meditation. The efficacy of prayer has been the topic of various scientific studies since Francis Galton first addressed it in 1872. In recent decades, studies on prayer have become notable in medicine. According to the Washington Post, (March 2006) "...prayer is the most common complement to mainstream medicine, far outpacing acupuncture, herbs, vitamins and other alternative remedies." Recent medical studies on prayer have generally shown mixed results when it comes to healing from illnesses (Carey 2006) ${ }^{[5]}$.

Since ages, Indians have believed in the power of chanting the sacred word OM, which is generally referred to as Hindu's religious symbol. For many of us, it may be just a word created out of our rich mythological past. But you will be amazed to know that even science has agreed with the therapeutic, psychological and spiritual benefits that you derive from chanting OM.

If you utter the word OM without using your tongue, you will feel a certain amount of vibration in your body. As per the Vedas, all the ordinary audible sounds such as sound we hear from bow and strings, waves against the shore, drum and stick, winds against the leaves, etc. only create when they strike each other creating waves of air molecules, 
which we perceive as sound. On the contrary, the sound of $\mathrm{OM}$ is not a result of vibration or striking of two objects. It evolves on its own. It is believed to be the primal sound of the universe that comprises of all the sounds within itself.

Over the time, the chanting of OM has popularized even in western cultures and has become an integral part of meditation. The sacred word has been proven to provide a certain degree of peace and purification to the mind and soul. From sages to yoga practitioners to monks, everyone chants this Sanskrit word for various benefits. The OM mantra has astounding benefits that can heal the stress accumulation in the minds of people living in this ultra modern and busy life and if you read these 9 benefits of chanting the powerful "OM" mantra, you will get motivated to try this OM therapy for yourself.

Medical studies have found that chanting OM with concentration enables you to reduce the adrenaline levels that in turn help in reducing the stress levels. Since chanting OM segregates you from the world and the associated tensions, you tend to find peace without the distraction and you feel piousand serene. Whenever, you feel upset or depressed, find a suitable place to meditate and chant OM SO that you get a chance to peek into yourself and your ideologies.

Our minds have become accustomed to attracting negative things in a way that we imagine negative consequences at first for any given situation. Even the law of attraction mentions that when we think more of negative things, we send negative vibes to the universe which is sent back to us in negative forms. But when you chant OM, you start concentrating on yourself and your soul that drives away the negativity and helps you in interacting with positive people only. You automatically start distinguishing between negative and positive things.

One of the most popular misconceptions about "OM" is that it is religious. On the contrary, it is the most universal syllable because it neither refers to any particular religion nor God. There have been several theories behind the OM mantra with the most popular explanation being that it was the cosmic sound which initiated the creation of universe (big bang theory). But that alone would not have been sufficient to turn it into a regular practice in Indian culture. In an experimental study Gurjar and Ladhake (2009) ${ }^{[10]}$ found that if one gently alloww the OM mantra to flow with the breath, the mind becomes calm. When the mind becomes calm, the body relaxes and the breath becomes even soother and slower.

That rate of speed at which the OM mantra is being repeated naturally slows down. It is not a matter of forcing the mantra to slow, but rather, this slowing comes quite naturally. Allow the mind to stay wide-awake and alert, as the OM mantra and breath become naturally slower and slower. Within minutes individual's mind and body begin to relax. By this analysis we could conclude stress gets minimized after OM chant (Gurjar and Ladhake, 2009) ${ }^{[10]}$. Stigsby et al. (1981) ${ }^{[25]}$ conducted a study on the effect of mantra meditation on the electroencephalograms of experienced mediators. The results were inconclusive. Seer and Raebum 4 conducted a similar study on the effect of meditation training on hypertension. Here also, the study showed modest reductions in blood pressure, but the results were again inconclusive.

Telles et al. (1994) ${ }^{[27]}$ conducted experiments on the effect of OM meditation on Middle Latency Auditory Evoked
Potentials of 18 male subjects between the ages of 25 and 45 years, 9 of who had more than 10 years of experience in OM meditation and the other 9 had no experience at all. The results indicated that the experimental group showed an increase in the peak amplitude of $\mathrm{Na}$ wave whereas there was a significant decrease in the control group. They extended this study in 1996 with the experimental group meditating on $\mathrm{OM}$ and the control group meditating on a neutral word one. Mental repetition of OM showed a significant decrease in skin resistance level of the experimental group as against the control group. There was also a reduction in the heart rate and the rate of breathing.

Takahashi et al. (1999) ${ }^{[26]}$ conducted a pilot study on the effect of low frequency noise on human body vibration. They showed that the low frequency noise affects the health of individuals depending on the structure of the body. The frequency range used by them was from 20 to $50 \mathrm{~Hz}$, which is quite below the frequency of a normal human voice.

Heisnam Jina Devi et al. (2004) ${ }^{[14]}$ conducted experiments to study signal characteristics of mantra sound patterns. They showed that OM(A-U-M) signal has two segments, starting with $\mathrm{O}$ and gradually tapering off to $\mathrm{M}$.

In a historical context, in 1571 Pope Pius V called for all of Europe to pray the Rosary for victory at the Battle of Lepanto, in which the Christian belligerents included the Papal States. Trophies from the battle are now enshrined in various Christian churches which attribute the victory to the massive prayers (Gilbert, 2004) ${ }^{[9]}$.

Prayer has been reported to improve outcomes in human as well as nonhuman species, to have no effect on outcomes, to worsen outcomes and to have retrospective healing effects (Andrade and Radhakrishnan, 2009) ${ }^{[14]}$.

\section{Method}

Sample: The sample consisted of high stressed 20 adult male and 10 adult female subjects, aged between 25 to 35 years, screened on the basis of Cohen's (1988) ${ }^{[6]}$ Perceived Stress scale. They were asked to recite OM word daily in the morning for 10 minutes with intention and devotion for 21 days in a green garden.

Research Tool: Cohen's (1988) ${ }^{[6]}$ Perceived Stress scale was used to assess the level of stress among the subjects.

Results: Mean perceived stress levels of subjects obtained on first test and the final test were compared through t-ratio statistical significant test, and the results were obtained as presented in Table-1.

Table 1: Comparison of Mean Perceived Stress Levels of Subjects obtained on First Test and the Final Test

\begin{tabular}{|c|c|c|c|c|c|c|c|}
\hline Condition & $\mathbf{N}$ & Mean & SD & r & t-ratio & df & p-value \\
\hline First Test & 30 & 20.62 & 4.03 & & & & \\
\hline & & & & -.76 & 12.17 & 29 & $<.01$ \\
\hline Second Test & 30 & 16.36 & 3.65 & & & & \\
\hline
\end{tabular}

It is evident from the contents of the Table- 1 that continuous reciting the OM word for 3 weeks had significantly reduced the stress level by $21.95 \%$ among the subjects. The obtained difference between the mean stress scores of first test (M$20.62+4.03)$ and the second test $(M=16.62+3.65)$ was significant $(\mathrm{t}=12.17$; df-29; $\mathrm{p}<.01)$ beyond chance, which confirms the hunch that reciting OM word isa significant antidote of stress. Kalyani et al (2011) ${ }^{[16]}$ in their study 
observed significant deactivation bilaterally during 'OM'chanting in comparison to the resting brain state in orbito-frontal, anterior cingulate, parahippocampal gyri thalami and hippocampi. In addition the right amygdala demonstrated significant deactivation. No significant activation was observed during 'OM' chanting. The use of 'OM' chanting for meditation is well known (Kumar et al., 2010) ${ }^{[20]}$ Effective 'OM' chanting is associated with the experience of vibration sensation around the ears. It is expected that such a sensation is also transmitted through the auricular branch of the vagus nerve.

\section{References}

1. Andersen J. Meditation meets behavioral medicine: The story of experimental research on meditation. J Consciousness Stud. 2000; 7:17-73.

2. Andrade Chittaranjan, Radhakrishnan Rajiv. Prayer and healing: A medical and scientific perspective on randomized controlled trials. Indian J Psychiatry. 2009; 51(4):247-253.

3. Bames VA, Davis HC, Murzynowski JB, Treiber FA. Impact of meditation on resting and ambulatory blood pressure and heart rate in youth. Psychosom Med. 2004; 66:909-14,

4. Anderson JW, Liu C, Bonadonna R. Meditation's impact on chronic illness. Holist Nurs Pract. 2003; 17:309-19.

5. Carey Benedict. Long-Awaited Medical Study Questions the Power of Prayer. New York Times, 2006. Retrieved 17 November 2013.

6. Cohen S. Perceived Stress Scale. Mind Garden, USA, 1988.

7. Cysarz D, B?ssing A. Cardiorespiratory synchronization during Zen meditation Eur J Appl Physiol. 2005; 95:88-95.

8. Davidson RJ, Kabat-Zinn J, Schumacher J, Rosenkranz M, Muller D, Santorelli SF. Alterations in brain and immune function produced by mindfulness meditation. Psychosom Med. 2003; 65:564-70.

9. Gilbert Chesterton. Lepanto, Ignatius Press, 2004. ISBN 1586170309

10. Gurjar AA, Ladhake Siddharth A. Spectral Analysis of Sanskrit Devine Sound OM. Information Technology Journal. 2009; 8:781-785.

11. Gurjar AA, Ladhake SA. Time-frequency analysis of chanting Sanskrit divine sound OM mantra. Int. J Comput. Sci. Network Secur. 2008; 8:170-175.

12. Gurjar AA, Ladhake SA, Thakare AP. Analysis of acoustic of OM chant to study it's effect on nervous system. Int. J. Comput. Sci. Network Sec. 2008; 9:363367.

13. Jain S, Shapiro SL, Swanick S, Roesch SC, Mills PJ, Bell I. A randomized controlled trial of mindfulness meditation versus relaxation training: Effects on distress, positive states of mind, rumination, and distraction. Ann Behav Med. 2007; 33:11-2111.

14. Jina Devi Heisnam, Swamy NVC, Nagendra HR. Spectral analysis of the Vedic mantra Omkara. Indian J Traditional Knowledge. 2004; 3:154-161.

15. John PG, Manolakis GD. Digital Signal Processing: Principles, Algorithms and Applications. 3rd Edn., Prentice Hall, New York, 1999.

16. Kalyani Bangalore G, Venkatasubramanian Ganesan, Arasappa, Rashmi Rao, Naren Sunil v Kalmady P,
Rishikesh Behere $\mathrm{V}$ et al. Neurohemodynamic correlates of $\mathrm{OM}^{\prime}$ chanting: A pilot functional magnetic resonance imaging study. Int J Yoga. 2011; 4(1):3-6.

17. Khalsa DS. Medical meditations. Total Health. 2000; 22:59-61.

18. Kjaer TW, Bertelsen C, Piccini P, Brooks D, Alving J, Lou HC. Increased dopamine tone during meditationinduced change of consciousness Brain Res Cogn Brain Res. 2002; 13:255-9.

19. Kryscio RJ. Blood pressure response to transcendental meditation: A meta-analysis. Am J Hypertens. 2008; 21:310-6.

20. Kumar S, Nagendra H, Manjunath N, Naveen K, Telles S. Meditation on 'OM: Relevance from ancient texts and contemporary science. Int J Yoga. 2010; 3:2-5.

21. Rabinar LR, Juang BH. Fundamentals of Speech Recognition (Prentice Hall Signal Processing Series). Prentice Hall PTR. USA., ISBN: 9780130151575, 1993, 507.

22. Researchers Look at Prayer and Healing 451 Religious Coping Strategies For.

23. Solberg EE, Ekeberg O, Holen A, Ingjer F, Sandvik L, Standal PA et al. Hemodynamic changes during long meditation. ApplPsychophysiol Biofeedback. 2004; 29:213-21.

24. Solberg EE, Holen A, Ekeberg O, Osterud B, Halvorsen $\mathrm{R}$, Sandvik L. The effects of long meditation on plasma melatonin and blood serotonin. Med SciMonit. 2004; 10:CR96-101.

25. Stigsby B, Rosenberg JC, Moth HB. Electroencephalographic findings during mantra meditation (Transcendental meditation). A controlled, quantitative study of experienced meditators. Electroencephalogr. Clin. Neurophysiol. 1981; 51:434442.

26. Takahashi Y, Yonekawa Y, Kanada K, Maeda S. A pilot study on the human body vibration induced by low frequency noise. Indust. Health. 1999; 37:28-35.

27. Telles S, Nagarathna R, Nagendra HR, Desiraju T. Alterations in auditory middle latency evoked potentials during meditation on a meaningful symbol OM. Int. J Neurosci. 1994; 76:87-93.

28. Van Wijk EP, Ludtke R, Van Wijk R. Differential effects of relaxation techniques on ultraweak photon emission. J Alter Complement Med. 2008; 14:241-50.

29. Wachholtz AB, Pargament KI. Is spirituality a critical ingredient of meditation? Comparing the effects of spiritual meditation, secular meditation, and relaxation on spiritual, psychological, cardiac, and pain outcomes. J Behav Med. 2005; 28:369-84.

30. Williams AL, Selwyn PA, Liberti L, Molde S, Njike VY, McCorkle R et al. A randomized controlled trial of meditation and massage effects on quality of life in people with late-stage disease: A pilot study. J Palliat Med. 200, Washington Post, March 24, 2006, 2005. 